\title{
Recent patent applications in biosensors
}

\begin{tabular}{|c|c|c|c|c|c|}
\hline Patent number & Description & Assignee & Inventor & $\begin{array}{c}\text { Priority } \\
\text { application date }\end{array}$ & Publication date \\
\hline WO 2012098758 & $\begin{array}{l}\text { A detection apparatus for a biosensor with } \\
\text { an optical prism incident surface and a } \\
\text { detection plate adhesion surface that are set } \\
\text { at a specific angle; useful in, e.g., the medical } \\
\text { field. }\end{array}$ & $\begin{array}{l}\text { National Institute of } \\
\text { Advanced Industrial } \\
\text { Science and Technology } \\
\text { (Tokyo) }\end{array}$ & $\begin{array}{l}\text { Akiyama S, } \\
\text { Fujimaki M, } \\
\text { Nagata K }\end{array}$ & $1 / 20 / 2011$ & $7 / 26 / 2012$ \\
\hline MX 2010012079 & $\begin{array}{l}\text { A regenerable biosensor for determining the } \\
\text { enzyme activity of protease. The biosensor is } \\
\text { provided with a resonator that is provided with } \\
\text { a quartz microbalance unit, where the quartz } \\
\text { microbalance unit is provided with a coercive } \\
\text { power of } 10,000-40,000 \text { units, ensuring a } \\
\text { simple and efficient biosensor. }\end{array}$ & $\begin{array}{l}\text { Autonomous University } \\
\text { of Baja California } \\
\text { (Mexicali, Mexico) }\end{array}$ & $\begin{array}{l}\text { Salas BV, } \\
\text { Stoytcheva MS, } \\
\text { Zlatev RK }\end{array}$ & $1 / 13 / 2011$ & 7/19/2012 \\
\hline WO 2012096162 & $\begin{array}{l}\text { A sensor chip for measuring properties of } \\
\text { substances, e.g. cells, comprising a diaphragm } \\
\text { provided with two different surfaces and } \\
\text { through-hole(s) formed between surfaces, } \\
\text { where a portion of the surfaces and through- } \\
\text { hole is covered with a silicon layer. }\end{array}$ & $\begin{array}{l}\text { Panasonic } \\
\text { (Osaka, Japan) }\end{array}$ & $\begin{array}{l}\text { Hashimotodani K, } \\
\text { Nakano Y, } \\
\text { Nakatani M, Oka T, } \\
\text { Ushio H, Yamada Y, } \\
\text { Yamamoto T }\end{array}$ & 1/13/2011 & 7/19/2012 \\
\hline US 20120178178 & $\begin{array}{l}\text { A biosensor cartridge, e.g., mass-based sensor, } \\
\text { optical sensor, electrical sensor, quartz crystal } \\
\text { microbalance, cantilever sensor and surface } \\
\text { acoustic wave sensor, for automating biosensor } \\
\text { processes to detect biological material from a } \\
\text { biological sample, e.g., blood. }\end{array}$ & $\begin{array}{l}\text { Samsung Electronics } \\
\text { (Suwon, S. Korea) }\end{array}$ & $\begin{array}{l}\text { Choi YS, Do Jae P, } \\
\text { Han KY, Kim SK, } \\
\text { Lee HJ, Lee JH, } \\
\text { Lee JN, Lee SS, } \\
\text { Lee YH }\end{array}$ & $1 / 6 / 2011$ & 7/12/2012 \\
\hline US 20120168306 & $\begin{array}{l}\text { A biosensor system for performing affinity- } \\
\text { based detection to detect an analyte in a } \\
\text { biological sample. Uses include but are not } \\
\text { limited to toxin, hormone, DNA strand, protein } \\
\text { and bacteria. }\end{array}$ & $\begin{array}{l}\text { University of Texas } \\
\text { System (Austin, TX, } \\
\text { USA) }\end{array}$ & $\begin{array}{l}\text { Hassibi A, Jang B, } \\
\text { Manickam A }\end{array}$ & 11/17/2008 & 7/5/2012 \\
\hline JP 2012122744 & $\begin{array}{l}\text { A method for manufacturing a biosensor } \\
\text { involving adhering an electrode layer and a } \\
\text { cover layer on the surface of a spacer layer by } \\
\text { hot melt adhesive. }\end{array}$ & $\begin{array}{l}\text { Murata Manufacturing } \\
\text { Co. (Kyoto, Japan) }\end{array}$ & Tanigawa M & $12 / 6 / 2010$ & 6/28/2012 \\
\hline KR 2012057808 & $\begin{array}{l}\text { A biosensor useful for detecting gonyautoxin } \\
\text { (GTX) from phytoplankton, comprising anti- } \\
\text { GTX antiserum separated from a combination } \\
\text { of protein and GTX from immunized mouse. }\end{array}$ & $\begin{array}{l}\text { Korea Ocean R\&D } \\
\text { Institute (Ansan, } \\
\text { S. Korea) }\end{array}$ & $\begin{array}{l}\text { Jae HL, Lee TK, } \\
\text { Man C }\end{array}$ & $11 / 29 / 2010$ & 6/7/2012 \\
\hline KR 2012057596 & $\begin{array}{l}\text { A sensibility diagnostic system comprising } \\
\text { a sensibility diagnostic chip equipped with } \\
\text { a biosensor, where the sensibility diagnostic } \\
\text { unit converts emotions into an emotional } \\
\text { quotient based on the received signals from } \\
\text { the subject. The system is useful for diagnosis } \\
\text { of sensibility, e.g., stress. }\end{array}$ & $\begin{array}{l}\text { Yonsei University } \\
\text { Industrial-Academic } \\
\text { Co-operative Foundation } \\
\text { (Seoul) }\end{array}$ & Hyo IJ, Jung HL & 12/30/2011 & $6 / 5 / 2012$ \\
\hline KR 2012055009 & $\begin{array}{l}\text { A biosensor useful for multiplexed diagnostics, } \\
\text { comprising a plane light source, specific } \\
\text { antibody, detection area, color charge-coupled } \\
\text { device camera and long-pass filter. }\end{array}$ & $\begin{array}{l}\text { Electronics and } \\
\text { Telecommunications } \\
\text { Research Institute } \\
\text { (Daejon, S. Korea) }\end{array}$ & $\begin{array}{l}\text { Bong PH, Moon YJ, } \\
\text { Park JW }\end{array}$ & $11 / 22 / 2010$ & $5 / 31 / 2012$ \\
\hline CN 102477416 & $\begin{array}{l}\text { A high-salt lipase useful in the printing, } \\
\text { petrochemical and environmental industries, } \\
\text { single-cell protein production, cosmetic } \\
\text { production, biological material, biosensor } \\
\text { and biomedical fields. The high-salt lipase is } \\
\text { cost effective and has strong selectivity and } \\
\text { specificity with improved enzyme activity, } \\
\text { stability and practicability. }\end{array}$ & Zhang B & $\begin{array}{l}\text { Fang J, Liu G, Su D, } \\
\text { Zhang B }\end{array}$ & 9/5/2011 & 5/30/2012 \\
\hline
\end{tabular}

Source: Thomson Scientific Search Service. The status of each application is slightly different from country to country. For further details, contact Thomson Scientific, 1800 Diagonal Road, Suite 250, Alexandria, Virginia 22314, USA. Tel: 1 (800) 337-9368 (http://www.thomson.com/scientific). 